\title{
More results on theories inside the conformal window
}

\section{Maria Paola Lombardo}

INFN-Laboratori Nazionali di Frascati

E-mail: mariapaola.lombardo@lnf.infn.it

\section{Kohtaroh Miura}

Kobayashi-Maskawa Institute for the Origin of Particles and the Universe, Nagoya University

E-mail: miura@kmi.nagoya-u.ac.jp

\section{Tiago Nunes da Silva*}

University of Groningen

E-mail: t.j.nunes@rug.nl

\section{Elisabetta Pallante}

University of Groningen

E-mail: e.pallante@rug.nl

\section{Nestor Subiron Montoro}

University of Groningen

E-mail: n.subiron.montorodstudent.rug.nl

We present an update of our study in progress on the spectrum and topology of the $S U(3)$ gauge theory with $N_{f}=12$ flavors in the fundamental representation. We are extending our previously published results with ensembles at larger volumes and smaller quark masses and refining our method of measuring the spectrum. Preliminary results are presented and agree with our previous conclusions that the theory has an infrared fixed point.

31st International Symposium on Lattice Field Theory LATTICE 2013

July 29 - August 3, 2013

Mainz, Germany

\footnotetext{
* Speaker.
} 


\section{Introduction}

Chiral symmetry restoration in the large- $N_{f}$ limit of QCD-like theories has been the subject of much interest in recent years. An especially hot topic of interest within the lattice community has been for a while the SU(3) gauge theory with $N_{f}=12$ flavors of fundamental fermions. Our first study of this theory showed evidence that the zero temperature theory lies in the conformal window, where chiral symmetry is restored [1]. This theory has been studied by several other groups, and while our results agree with many of them, a few have presented contrasting views. A short list of references on twelve-flavor QCD includes but it is definitely not limited to [2, 3, 4, 5, 6, 7, 8, 9].

In recent works [10] we have focused on the nature of the strong coupling chiral symmetry breaking transition in this system and the effects of improvement. We are now conducting simulations to extend and improve on our previously reported results for the particle spectrum of this theory [1, 11]. In particular, we are adding ensembles with larger volumes and smaller quark masses and implementing a more refined method to obtain the hadron correlators. We are also studying the eigenvalue spectrum of the Dirac operator and the topology of the system, which can provide interesting hints for a complete understanding of the conformal window.

In Section 2 we briefly discuss the setup of our configuration generation. This will be followed by a discussion of our spectrum measurements and some preliminary results in Section 3, and the study of the Dirac spectrum in Section 4. Discussion and outlook are in Section 5. While most results presented here are preliminary, we expect to report soon on final results in upcoming works.

\section{Lattice setup}

We use the publicly available MILC suite to simulate the SU(3) gauge theory with $N_{f}=$ 12 degenerate flavors of staggered fundamental fermions on the IBM BG/P in Groningen. The configurations are generated using the tree-level Symanzik improved gauge action. Improvement is extended to the fermion action following the Naik prescription. We are running a range of lattice quark masses going from $a m=0.01$ to $a m=0.07$ at fixed coupling $\beta=3.9$. For the heaviest masses $a m=0.06$ and $a m=0.07$ the volumes $16 \times 24$ and $24^{4}$ are being simulated. For the remaining lighter masses volumes $24^{4}$ and $32^{4}$ are being simulated.

In [10] we have shown that, as an effect of improvement, and as one goes from weak to strong coupling (in our case for masses $a m \lesssim 0.04$ ) the theory undergoes a phase transition to a genuine lattice artifact intermediate phase, before a chiral symmetry breaking bulk transition takes place. The choice $\beta=3.9$, which was already used in previous studies by our group, ensures that these simulations are carried out in the chirally symmetric phase and sufficiently far from artifacts.

Measurements of observables such as the chiral condensate and the plaquette are performed on the fly, while configurations are stored every two molecular dynamics time units well after thermalization for spectrum and topology measurements.

\section{Hadron Spectrum}

We are measuring the hadron spectrum on our saved configurations with different types of sources. The preliminary results presented in this work have been obtained with fixed Coulomb 
gauge corner-wall sources. This procedure effectively amounts to a source smearing that reduces contamination from excited states, better isolating the ground state of the system.

We also found it useful in our study to construct the hadron correlators from quark propagators with different combinations of boundary conditions in the time direction. As also shown in [12] and explicitly implement in [8], if $\overline{C(t)}=(1 / 2)\left[C_{\text {p.b.c. }}(t)+C_{\text {a.b.c. }}(t)\right]$ is the average of hadron correlators with periodic (p.b.c.) and antiperiodic (a.b.c.) boundary conditions on the time interval of size $\mathrm{T}$, then it may be written as the average $\overline{C_{\text {p.b.c. }}}(t)$ with periodicity $2 T$. In other words, the resulting combined hadron correlators will have their lattice temporal extent effectively doubled. This is exemplified in Figure 1. The periodic and anti periodic hadron correlators, $C_{\text {p.b.c. }}(t)$ and $C_{\text {a.b.c. }}(t)$, are built as averages over all possible products of periodic and anti periodic quark propagators.

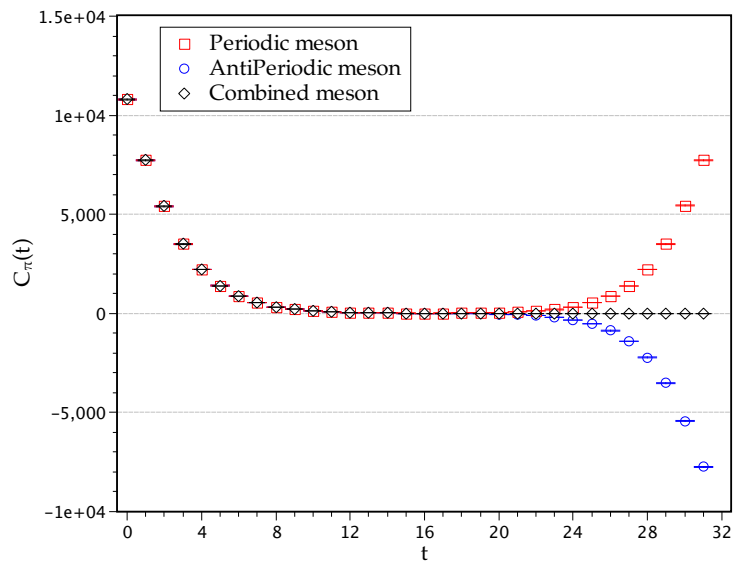

(a)

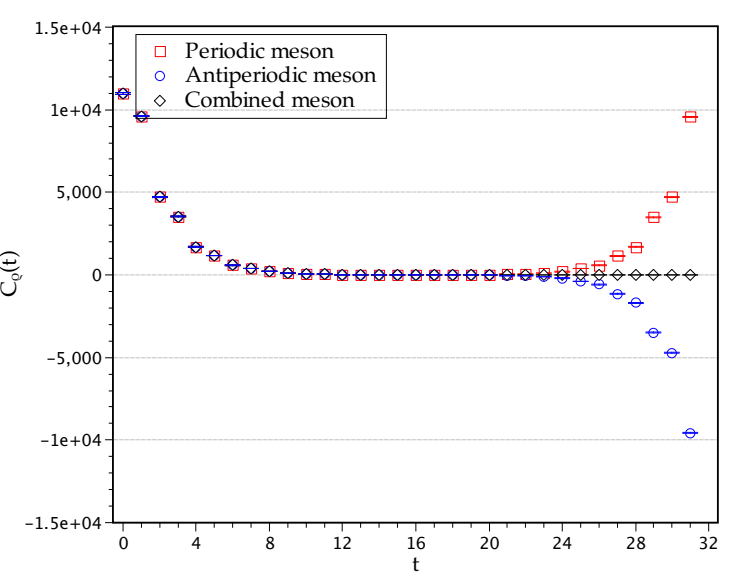

(b)

Figure 1: We show samples of antiperiodic and periodic meson correlators obtained from configurations generated with bare quark mass $a m=0.05$. (a) The pseudoscalar meson. (b) The vector meson.

As also suggested in [8], in the case of the pion and vector correlators it may be useful to consider the combination

$$
\tilde{C}(2 t)=\frac{C(2 t)}{2}+\frac{C(2 t-1)}{4}+\frac{C(2 t+1)}{4}
$$

in order to suppress the constant oscillation mode that might appear in the correlators (and increasing toward smaller masses at fixed $\beta$ ) as a result of the wrapping of a single quark line around the antiperiodic time direction. In all analyzed cases we are able to clearly identify the effective mass plateaus for the fundamental state. We report here on some preliminary results. The analysis of the correlators for the lightest quark mass is still ongoing and the results presented here correspond to a small subset of our ensemble, therefore being very preliminary. The analysis of this full ensemble together with a complete study of finite volume effects will be presented in a forthcoming paper.

First we look at the ratio $m_{\pi} / m_{\rho}$. In a theory with spontaneous chiral symmetry breaking the pion is a Goldstone boson of the broken symmetry and scales as $m_{\pi} \sim \sqrt{m}$. The vector mass instead is given by a constant term plus a leading correction which is linear in the quark mass $m_{\rho} \sim m_{\rho}^{0}+C m$. Thus the pion to vector mass ratio would behave as $m_{\pi} / m_{\rho} \sim \sqrt{m}$ in the broken phase and would extrapolate to zero in the chiral limit. If chiral symmetry is restored, on the other hand, all hadron masses would scale as power laws with the quark mass with roughly the same 
exponent (and exactly the same at the infrared fixed point) so that the ratio $m_{\pi} / m_{\rho}$ should remain constant to a good approximation as the quark mass varies. This latter scenario is observed in our simulations, favoring chiral symmetry restoration.

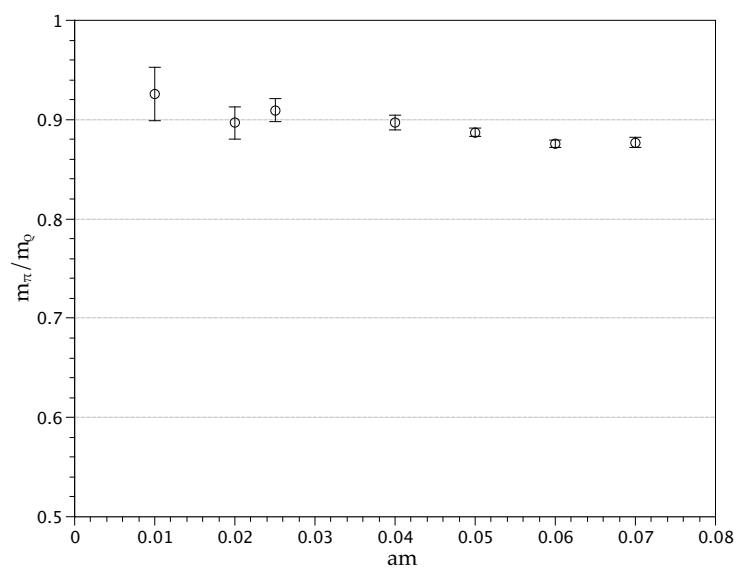

(a)

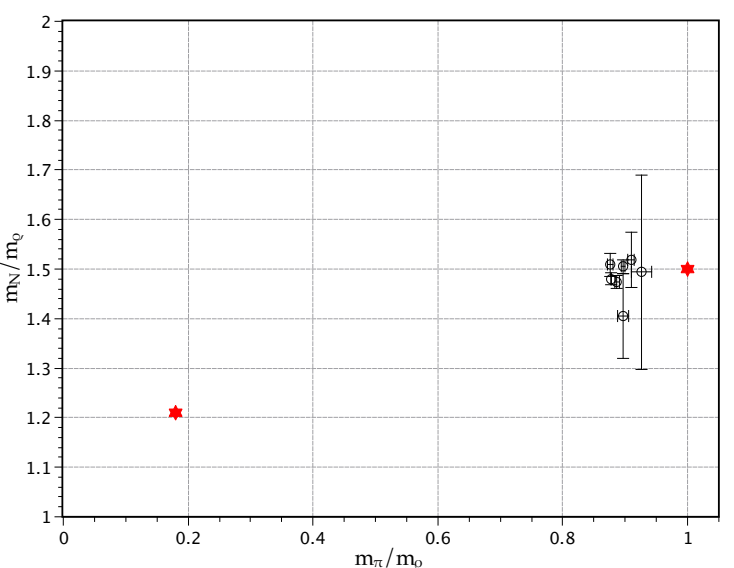

(b)

Figure 2: (a) Ratio $m_{\pi} / m_{\rho}$ as a function of the bare quark mass. No scaling towards zero is observed in the chiral limit. (b) The Edinburgh plot for the updated ensemble of hadron correlators. The leftmost red point represents the physical point of QCD $\left(m_{\pi} / m_{\rho} \simeq 0.18\right.$ and $\left.m_{N} / m_{\rho} \simeq 1.21\right)$, while the rightmost red point represents the heavy quark limit $\left(m_{\pi} / m_{\rho} \simeq 1\right.$ and $\left.m_{N} / m_{\rho} \simeq 3 / 2\right)$. The clustering of points for the whole range of simulated quark masses is a signal of chiraly symmetry restoration. In both figures the point at $a m=0.01$ is preliminary, with limited statistics.

Another interesting way of looking at the system is inherited from QCD and was first used in the study of the conformal window in [11]; it uses the well known Edinburgh plot depicted in Figure 2(b). In a chirally broken phase one expects the ratios of hadron masses to scale from the heavy quark limit point towards the physical point as the quark mass is decreased. In contrast, if the theory exhibits restored chiral symmetry, the ratios of hadron masses should cluster around a single point of the Edinburgh plot for all quark masses, its location being dictated by the anomalous mass dimension of the theory. The results observed in our simulations show this clustering at $m_{\pi} / m_{\rho}<1$ and for a range of lattice masses between $a m=0.01$ and $a m=0.07$ suggesting again that the theory has exact chiral symmetry and it is inside the conformal window.

\section{Topology}

We are also conducting a study of the topology of the system. To measure the topological charge we smooth the gauge fields with APE smearing steps. We measure the topological charge before and during the smearing every 10 sweeps, in order to monitor the evolution. With a limited statistics of about 200 configurations for each given pair of mass and volume (configurations are saved and measured every two molecular dynamics time units) we never observe a non-smoothed topological charge significantly deviating from zero. The same result is true for both hot and cold starts in the Monte Carlo history. It is known that the autocorrelation within a single Markov chain tends to be much larger for topological observables than for other quantities, requiring a more 
accurate numerical study in the case of ordinary QCD. However, we should expect the tunneling probability to non-zero topological charge sectors to be highly suppressed for large- $N_{f}$ QCD-like theories inside the conformal window - where chiral symmetry is exact. In a forthcoming paper we plan to extensively discuss the theoretical implications for topology in the case of a theory inside the conformal window, and provide a more extensive supporting numerical analysis.

Here, we observe that the measured value of the topological charge starts very close to zero and typically stabilizes at zero value after about 50 -100 APE smearing steps, as shown in Figure 3(a). It is also true that the topological charge of a gluonic configuration in not uniquely defined at finite lattice spacing, thus measurements with different actions and cooling procedures are being carried out to check for consistency. In Figures 4 and 5 we present the results obtained from the first 250 low-lying eigenvalues of the Dirac operator for quark mass $a m=0.01$ and volume $32^{4}$.

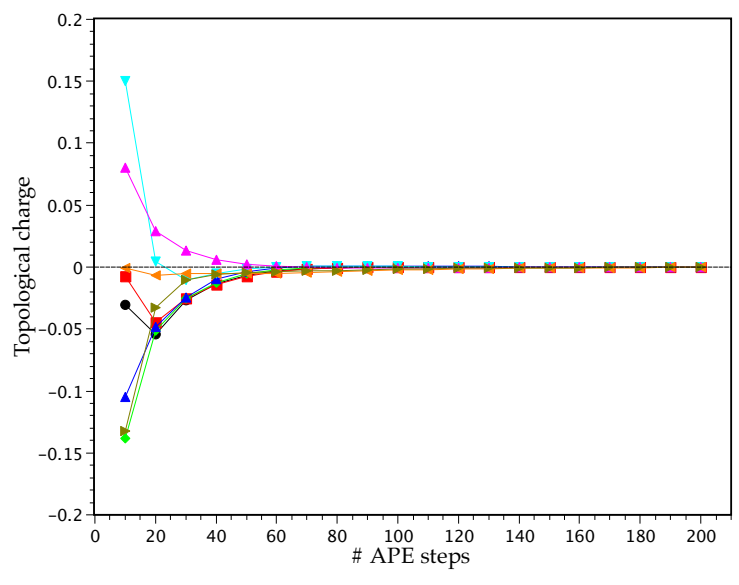

(a)

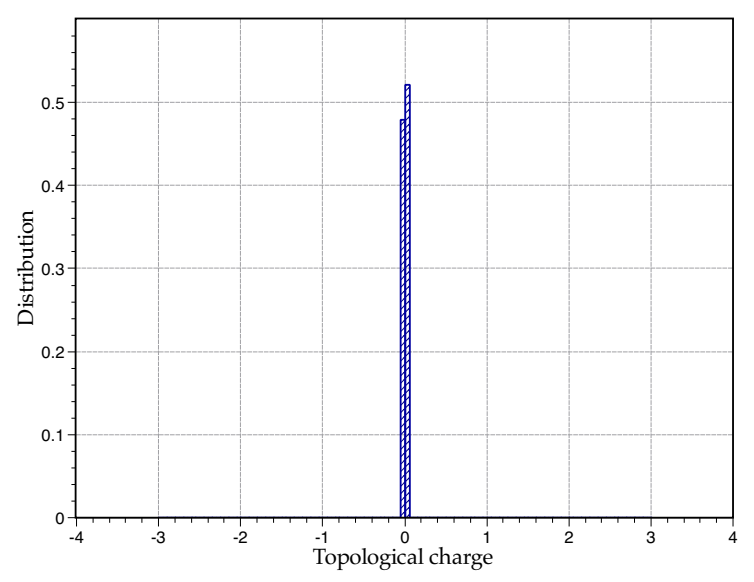

(b)

Figure 3: (a) Evolution of the measured topological charge for some representative configurations of the $\mathrm{am}=0.01$ ensemble with volume $32^{4}$. (b) Distribution of the measured topological charge. Results indicate that the system is in the zero topological charge sector.

For illustration purposes, and to better test the exact chiral symmetry hypothesis, we measure the eigenvalues of the Dirac operator and their chirality both for the thermalized but not smeared configurations, and after APE smearing. As can be seen in Figure 4(a), APE smearing helps in reducing taste breaking as expected and makes it possible to identify the degeneracy into quartets of the eigenvalues of the staggered Dirac operator. As expected, the tree-level Naik improved staggered fermion action used in this study generally provides a smaller suppression of taste breaking than the $n-H Y P$ smeared or HISQ improved actions.

The evolution with APE sweeps of the eigenvalues and their chirality makes it possible to observe some interesting qualitative features of the system. Smearing pushes the entire eigenvalue spectrum upwards, away from zero (see Figure 4(a)). If zero modes associated with chiral symmetry breaking were present they would be pushed toward zero, while we observe the opposite (see also Figure 4(b)). In addition, the measured chirality for the first low-lying modes - already small before smearing - is further suppressed, as shown in Figure 4(b) and Figure 5(a). We observe no gap separating a region of small chirality at large eigenvalue number from a region of large chirality at small eigenvalue number, as would be expected in a system where chiral symmetry is broken. 
The eigenvalue density extracted from the first 250 eigenvalues measured on the thermalized non

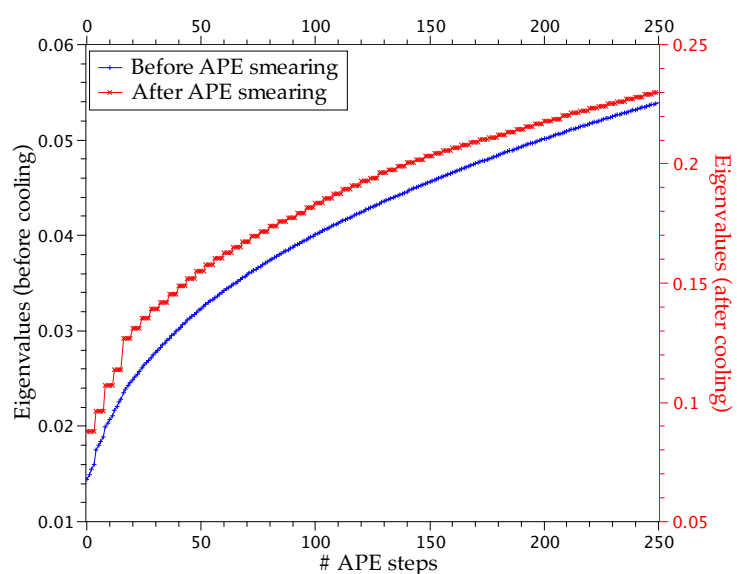

(a)

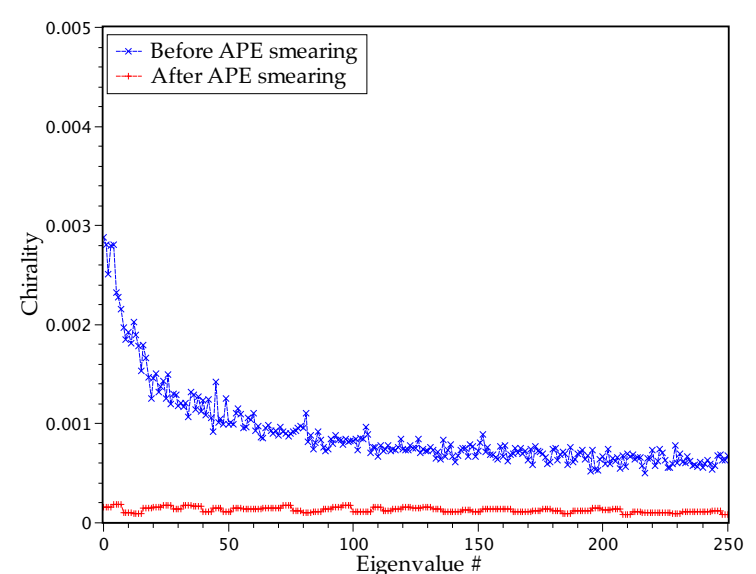

(b)

Figure 4: Effect of smearing on the measured eigenvalues (a) and their chirality (b). As expected, smearing in (a) reduces taste breaking and makes the staggered taste-quartets visible. Chirality of all eigenvalues is further suppressed by smearing, consistently with the behavior of a system in the chirally symmetric phase.

APE-smeared configurations for $a m=0.01$ and volume $32^{4}$ is shown in Figure $5(\mathrm{~b})$ and favors a zero extrapolated value of the eigenvalue density. Assuming to be in the regime where the BanksCasher relation can be applied, one would infer a zero chiral condensate at infinite volume and in the chiral limit, consistently with the direct determination of the chiral condensate in [1] and with the hypothesis of exact chiral symmetry.

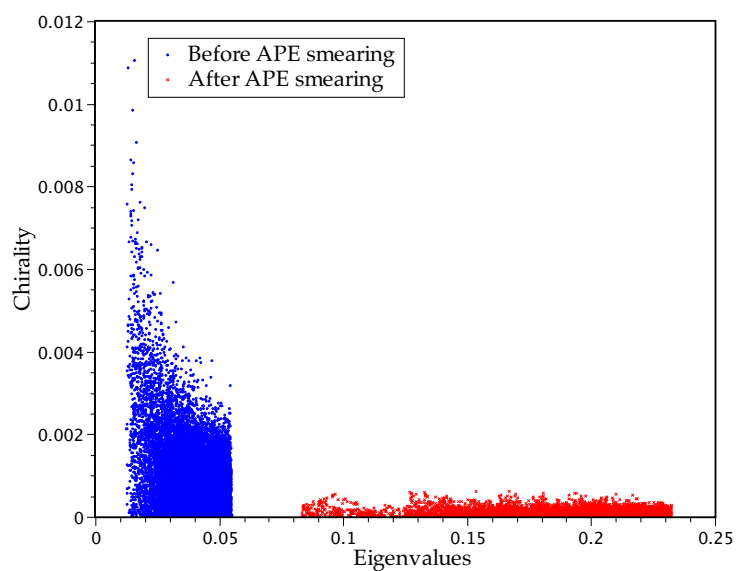

(a)

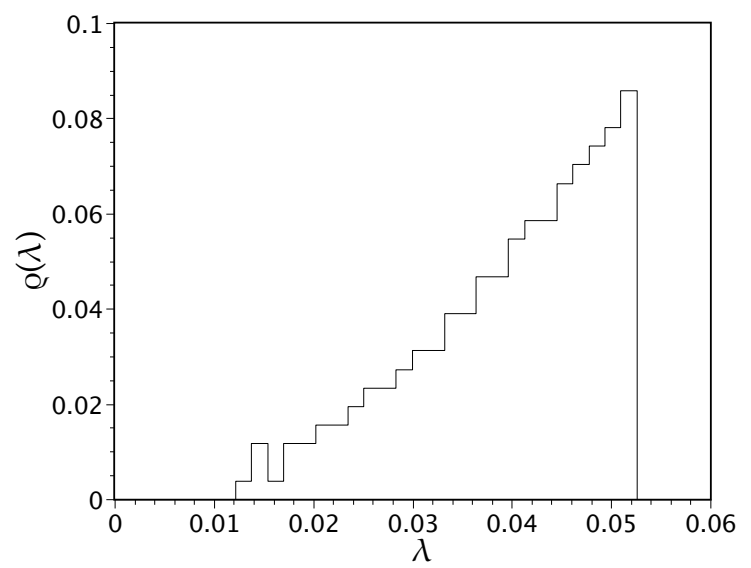

(b)

Figure 5: (a) Chirality as a function of the eigenvalues before and after APE smearing. The smearing pushes the eigenvalues to higher values and suppresses chirality even further. (b) Eigenvalue density measured at quark mass am=0.01 and volume $32^{4}$ on the non-smeared configurations.

\section{Conclusion and outlook}

We have reported on preliminary results of our study in progress on the spectrum and topology 
of the $S U$ (3) gauge theory with $N_{f}=12$ fundamental flavors on the lattice. We are extending our previously published results by adding new ensembles at larger volumes and smaller masses. We are also implementing a more refined method to measure the spectrum of these ensembles which rely on the combination of correlators with different boundary conditions on the time direction. Our preliminary results on the spectrum show no scaling to zero of the pion to vector ratio when approaching the chiral limit, nor scaling towards the physical QCD point in the Edinburgh plot. Both evidences support a scenario in which chiral symmetry is restored. On topology, we observe our configurations to be in the zero topological charge sector. We observe no high chirality modes for the first low-lying eigenvalues of the Dirac spectrum, suggesting that the index theorem is verified. Furthermore, smearing of the gauge fields pushes the eigenvalues further away from zero and chirality is even more suppressed. Both behaviors are again in agreement with a chirally restored scenario. Final results will be published soon in a more comprehensive work.

\section{Acknowledgments}

This work is in part based on the MILC collaboration's public lattice gauge theory code. The authors thank Vincent Thiery for contributions in the early stage of this work. Computing time was provided through the Dutch National Computing Foundation (NCF) and the University of Groninen.

\section{References}

[1] A. Deuzeman, M. P. Lombardo and E. Pallante, Phys. Rev. D 82 (2010) 074503 [arXiv:0904.4662 [hep-ph]].

[2] Z. Fodor, K. Holland, J. Kuti, D. Nogradi, C. Schroeder, K. Holland, J. Kuti and D. Nogradi et al., Phys. Lett. B 703 (2011) 348 [arXiv:1104.3124 [hep-lat]].

[3] T. Appelquist, G. T. Fleming, M. F. Lin, E. T. Neil and D. A. Schaich, Phys. Rev. D 84 (2011) 054501 [arXiv:1106.2148 [hep-lat]].

[4] T. DeGrand, Phys. Rev. D 84 (2011) 116901 [arXiv:1109.1237 [hep-lat]].

[5] P. de Forcrand, S. Kim and W. Unger, JHEP 1302 (2013) 051 [arXiv:1208.2148 [hep-lat]].

[6] X. -Y. Jin and R. D. Mawhinney, PoS LATTICE 2011 (2011) 066 [arXiv:1203.5855 [hep-lat]].

[7] A. Cheng, A. Hasenfratz, G. Petropoulos and D. Schaich, JHEP 1307 (2013) 061 [arXiv:1301.1355 [hep-lat]].

[8] Y. Aoki, T. Aoyama, M. Kurachi, T. Maskawa, K. -i. Nagai, H. Ohki, A. Shibata and K. Yamawaki et al., Phys. Rev. D 86 (2012) 054506 [arXiv:1207.3060 [hep-lat]].

[9] E. Itou, PTEP 2013 (2013) 8, 083B01 [arXiv:1212.1353].

[10] A. Deuzeman, M. P. Lombardo, T. Nunes Da Silva and E. Pallante, Phys. Lett. B 720 (2013) 358 [arXiv:1209.5720 [hep-lat]].

[11] A. Deuzeman, M. P. Lombardo and E. Pallante, PoS LATTICE 2011 (2011) 083 [arXiv:1201.1863 [hep-lat]].

[12] K. Sasaki and S. Sasaki, Phys. Rev. D 72 (2005) 034502 [hep-lat/0503026]. 